\title{
Introducing a chest pain pathway in the emergency department to improve quality of care for patients with possible cardiac chest pain
}

Jill Byrne

Belfast Health and Social Care Trust

\begin{abstract}
Chest pain is a common reason for patients to present to an emergency department (ED). It is crucial not to miss presentations of the potentially life-threatening acute coronary syndrome (ACS), although often these people present with a non-diagnostic ECG. This makes recognition of a history consistent with ACS very important. We noted inconsistencies in assessment, with many admissions to cardiology beds who did not prove to have ACS and some erroneous discharges who subsequently did have an ACS.
\end{abstract}

We introduced a history based risk tool as part of a chest pain pathway into the ED for use by medical staff assessing patients presenting with chest pain. The intervention involved a nurse from cardiology engaging with clerical, nursing, and medical staff in the ED to ensure success of this quality improvement project.

The project showed a reduction in admissions to cardiology with suspected ACS from $29 \%$ to $15 \%$, with a projected saving of £889 per patient who was prevented from being admitted. In addition, admissions became more appropriate, with an increase in the proportion of patients with a final diagnosis of ACS from $25 \%$ to $46 \%$ and a reduction in admissions with atypical chest pain from $75 \%$ to $54 \%$.

\section{Problem}

Many patients present to the emergency department with chest pain. It is important to identify those patients with acute coronary syndromes (unstable angina, acute myocardial infarction, or evolving infarction). However, several problems exist in identification of these patients. Up to $60 \%$ of patients with ACS may present with a non-diagnostic ECG.[1] Alternatively, ECG changes may be missed.[2,3]

Many patients are admitted with suspected ACS, although prevalence is only $8-10 \%$ in an ED chest pain population. [4] This causes the blocking of cardiology beds with many inappropriate admissions, and some patients can be missed, particularly if ECG changes are not noted or the patient presents with unstable angina.[5-7] If a patient presents with increasing chest pain on exertion at progressively lower workload, but without pain at rest, this represents prodromal angina but may be mistaken as stable angina.[8] Therefore, these patients could be inappropriately discharged when they may be in fact at risk of myocardial infarction.

\section{Background}

The European Society of Cardiology [8] define ACS as a clinical spectrum of disease, ranging from unstable angina (no myocardial damage but increased risk of myocardial infarction and subsequent complications), non-ST elevation myocardial infarction (NSTEMI some damage to myocardium), and ST elevation myocardial infarction (STEMI) which reflects a completely occluded coronary artery.
Diagnosis is made on the presence of an ischaemic ECG, suggestive clinical history, or the presence of circulating biomarkers of myocardial necrosis (Troponin). Two out of these three factors must be present to reach a diagnosis of myocardial infarction.[9] However, forty to sixty per cent of people who are subsequently diagnosed with ACS present to the ED with an initial non-diagnostic ECG. $[1,10]$ Some of these patients will not have raised troponin initially, and those with unstable angina will not have elevated troponin at any point, unless they go on to develop an MI. This means that the only way these people can initially be recognised is through the history of the presenting complaint. The full extent of coronary artery disease is often not clear until the patient undergoes invasive investigations such as coronary angiography.[8]

Difficulties in recognition of ACS and the subsequent inconsistencies in management of these patients are well known and challenge clinicians worldwide.[2-5] DeBusk et al [11] report that half of all patients presenting with chest pain are hospitalised to detect the ten per cent who have an ACS, but that this high admission rate still fails to identify the small percentage of patients with ACS that are discharged inappropriately.

Some tools are available for use in the ED [12-16], but many of these suppose knowledge of the various presentations of ACS [16], are based on populations with confirmed ACS [14,15], or are aimed at recognition of $\mathrm{MI}$ but not unstable angina $[12,13]$. The Goldman score[12], TIMI score [14], GRACE score [15], and HEART scores [16] were all trialled and found to be unhelpful. This is mainly because Goldman is outdated and does not have any criteria relating to unstable angina, which we did not want to miss. TIMI and GRACE were validated on cohorts of patients with confirmed ACS and rely on clinical parameters which do not relate to the presenting 
complaint, such as createnine and systolic blood pressure. We found that patients who were over 80 years with renal impairment and high systolic blood pressure were likely to be admitted whatever the nature of the chest pain.

As a result, a history based tool built on the internationally agreed clinical features of ACS $[8,17]$ was devised and integrated into a pathway for use in the ED to help aid recognition of ACS and standardise care. The rationale for the use of this tool was discussed during medical teaching.

See supplementary file: ds3253.pdf - "Pathway for diss"

\section{Baseline measurement}

Evidence that there were inappropriate admissions and discharges from the group of patients presenting to the ED with chest pain was anecdotal. Data from the coding and information department was found to contain duplication of data and gave no information on whether discharges were appropriate or not. For this reason, the data was searched by hand. ED patient documentation on 500 attendances was audited. Patient identification data, ECG data, whether the patient was admitted, or discharged was collected at baseline. Admitted patients were followed up to assess discharge diagnosis. Outcome measures were set at admission rate with possible ACS from the ED, incidence of confirmed ACS in admitted patients and whether any patients were inappropriately discharged. This was decided by assessment of the initial ECG and history and by whether the patient reattended with a subsequent diagnosis of ACS. Baseline measurement showed an admission rate of $29 \%$ (145/500), a rate of definite ACS of $25 \%(36 / 145)$ and inappropriate discharge rate of $5 \%$ (2/38 ACS were allowed home inappropriately).

\section{Design}

A project nurse from cardiology was appointed to a six month secondment to try to address inconsistencies in assessment and management.

Teaching for the junior medical staff on chest pain, ACS, and ECG interpretation were introduced at the beginning of induction and ad hoc as required.

Inconsistencies in patient assessment also needed to be addressed. One solution was the creation of a team of senior cardiology nurses to fast-track patients through the system. This was an attractive option, but unfortunately there was no funding for training, study leave, and shadowing. Despite organising in-house training, interest dwindled since no leave was available for competency development and team members had to use up days off and annual leave, and, although they were keen to develop, the potential team members felt that adequate opportunity was not provided.

In organising a nurse-led team, the idea of using a pathway to direct care through the ED had been discussed. The GRACE score, TIMI score, and Goldman scores were all trialled and found to be unhelpful. This is mainly because Goldman is outdated and does not have any criteria relating to unstable angina, which we did not want to miss. TIMI and GRACE were validated on cohorts of patients with confirmed ACS and rely on clinical parameters which do not relate to the presenting complaint such as createnine and systolic blood pressure. We found that patients who were over 80 years with renal impairment and a high systolic blood pressure were scoring highly whatever the nature of the chest pain. For this reason, a history based tool was devised in collaboration between two cardiologists, two emergency physicians, and the project nurse. This tool has since been subject to further evaluation and a multicentre prospective validation study is planned.

The pathway was approved by ED and cardiology physicians and was introduced to standardise assessment of patients with chest pain following erroneous discharges of two patients with $\mathrm{Ml}$ overnight by junior medical staff. There had been resistance from senior ED medical staff to introduction of the pathway for use by medical staff prior to this. The pathway was supposed $t$ be used in assessment of the patient, with the doctor physically ticking off the boxes to assess whether the history was suggestive of ACS. The tool was devised to ensure patients with myocardial infarction and unstable angina would be identified from aspects of the history if ECG changes were not evident, or were missed.

Implementation proved challenging as the senior medical team, although agreeable to implementation of the pathway for junior staff, either did not use the pathway, or did not advocate or insist on its use by the junior staff.

\section{Strategy}

PDSA 1 was focused on training and implementing a team of senior cardiology nurses to address inconsistencies in assessment. This was abandoned due to lack of dedicated time for training and the fact that there was no funding.

PDSA 2 was focused on implementation of the chest pain pathway in the ED for junior doctors. The intervention was only partially successful due to inadequate engagement by senior medical staff and nursing staff within the ED. The pathway was not being placed with the ED documentation by nursing staff for use by medical staff and senior medical staff were not encouraging use. Therefore, while the project nurse was role-modelling and encouraging use, others were ambivalent about the project.

PDSA 3 followed increased efforts in engaging all ED staff in helping promote pathway use and physically ensure the pathway was present in the notes and available for use. This involved clerical staff handing the pathway to the patient at reception. The triage nurse was then responsible for ensuring it was present with the documentation once the patient was triaged. Data on numbers of admissions, and the proportions who were diagnosed with definite ACS were fed back to the senior medical staff in the ED. An audit on outcomes of patients admitted with evidence of pathway use was presented and appeared to show better results in terms of an outcome of definite ACS than unstructured assessment. This 
seemed to help persuade senior medical staff to advocate use to junior medical staff, and use improved.

See supplementary file: ds3260.docx - "pdsa 1"

\section{Post-measurement}

Data collection focused on percentage of patients admitted with evidence of pathway use. Initial results showed usage of the pathway in around $30 \%$ of admissions. It was noted that the history based tool was primarily used in patients with a non-diagnostic ECG and was not used so often in patients with a diagnostic ECG. Following PDSA cycle 3, use was measured at $69 \%$.

The admission rate was calculated from a sample of 500 consecutive attendances with possible cardiac chest pain, just as at the beginning of the project. The admission rate had reduced from $29 \%$ to $15 \%$.

The proportion of patients who were formally admitted with suspected ACS and who had a discharge diagnosis of definite ACS was found to be low, at $25 \%$ pre-intervention. The post-pathway proportion of patients with confirmed ACS was found to be $46 \%$, which was a big improvement.

Erroneous discharge was another outcome measure; two patients had been discharged erroneously in the pre-intervention sample of 500 attendances with possible cardiac chest pain. There were none in the post-intervention sample.

\section{Lessons and limitations}

The biggest learning point from this project was the importance of stakeholder engagement. Nursing staff, clerical staff, senior and junior medical staff all had to be engaged for the project to have any chance of success. Key to this was the communication of any successes associated with the intervention, and ensuring all staff felt that they were a part of the success.

A learning point has been that continuous monitoring with action taken as required is necessary to ensure sustained quality improvement.

Admissions, discharges, and pathway use are now monitored and recorded as part of the cardiac nurse practitioner's (previously project nurse) daily work. This enables action to be taken rapidly if required and allows for monthly reporting back to stakeholders.

\section{Conclusion}

Feedback from pathway users has been encouraged and welcomed. The feedback received has resulted in changes in wording when appropriate. It has been interesting to see that the history based tool has been quite successful in identification of patients with unstable angina and NSTEMI. It has been interesting to hear junior doctors say "I had to admit because the pathway said so" and then to discover the patient, in fact, had an ACS diagnosed.
It was very satisfying to see the success of this project in terms of standardising assessment, ensuring more appropriate admissions in terms of patients with a final diagnosis of cardiac chest pain and a total cessation of erroneous discharges. We have learned that audit must be continuous to ensure sustained quality improvement. Any issues can then be dealt with immediately.

\section{References}

1. Patel J, Gupta R, Roe T et al. Influence of presentation electrocardiographic findings on the treatment and outcomes of patients with non ST segment elevation myocardial infarction. Am J Cardiol 2014;113: 256-61.

2. Masoudi F, Magid D, Vinson D et al. Results of the Implications of the Failure to Identify High-Risk Electrocardiogram Findings for Emergency Department Quality in Myocardial Infarction (EDQMI) Study the Quality of Care of Patients With Acute Myocardial Infarction. Circulation 2006;114: 1565-71.

3. Berger J, Eisen L, Nozad V et al. Competency in electrocardiogram interpretation among internal medicine and emergency medicine residents. Am J Med 2005;118: 873-80.

4. Body R. Emergent diagnosis of acute coronary syndromes: Today's challenges and tomorrow's possibilities.

Resuscitation 2008;79: 41-5.

5. Sequist T, Bates D, Cook, E et al. Prediction of missed myocardial infarction among symptomatic patients without coronary heart disease. Am Heart J 2005;149: 74-81.

6. Pope J, Aufderheide T, Ruthazer R et al. Missed diagnoses of acute cardiac ischaemia in the emergency department. NEJM 2000;342: 1163-70.

7. Jones ID, Slovis CM. Pitfalls in evaluating the low-risk chest pain patient. Emerg Med Clin North Am 2010;28: 183-201.

8. Erhardt L, Herlitz J, Bossaert L et al. ESC Guidelines for the management of acute coronary syndromes in patients presenting without persistent ST-segment elevation. Eur Heart J 2011;32: 2999-3054.

9. Thygesen K, Alpert JS, Jaffe AS et al. Third universal de?nition of myocardial infarction. Eur Heart J 2012;33: 2551-67.

10. Hamm C, Bassand J, Agewall S et al. Chest pain (management of). Eur Heart J 2002;23: 1153-76.

11. DeBusk R, Miller N, Raby $L$ et al. Technical feasibility of an online decision support system for Acute Coronary Syndromes. Circ Outcomes 2010;3: 694-700.

12. Goldman L, Cook E, Johnson P et al. Prediction of the need for intensive care in patients who come to emergency departments with acute chest pain. NEJM 1996;331: 1498-1504.

13. Selker $\mathrm{H}$, Zalenski R, Antman E et al. An Evaluation of Technologies for Identifying Acute Cardiac Ischemia in the Emergency Department: Executive Summary of a National Heart Attack Alert Program Working Group Report. Ann Emerg Med 1997;29: 1-12.

14. Antman E, Cohen M, Bernink P et al. The TIMI risk score for unstable angina/non-ST elevation MI: A method for prognostication and therapeutic decision making. JAMA 


\section{BMJ Quality Improvement Reports}

2000;284: 835-42.

15. Fox K, Dabous O, Goldberg R et al. Prediction of death and myocardial infarction in the six months after presentation with acute coronary syndrome: prospective multinational observational study (GRACE) BMJ 2006;333: 1091-94.

16. Six AJ, Backus BE, Kelder JE et al. Chest pain in the emergency room: value of the HEART score. Neth Heart J 2008;16: 191-6.

17. National Institute for Clinical Excellence Clinical Guideline 95: Chest pain of recent onset: Assessment and diagnosis of recent onset chest pain or discomfort of suspected cardiac origin

http://www.nice.org.uk/nicemedia/live/12947/47931/47931.p df 2010 accessed 23/11/12.

\section{Declaration of interests}

No competing interests to declare.

\section{Acknowledgements}

Dr Brian McClements, Dr Suzanne Maynard, Dr Paul Curran, and Dr John Gray. 